\title{
Chern-Simons Field Theories with Non-semisimple Gauge Group of Symmetry
}

\author{
Franco Ferrari \\ Institute of Physics, University of Szczecin, ul. Wielkopolska 15, \\ 70-451 Szczecin, Poland:
}

November 1, 2018

\begin{abstract}
Subject of this work is a class of Chern-Simons field theories with nonsemisimple gauge group, which may well be considered as the most straightforward generalization of an Abelian Chern-Simons field theory. As a matter of fact these theories, which are characterized by a non-semisimple group of gauge symmetry, have cubic interactions like those of non-abelian Chern-Simons field theories, but are free from radiative corrections. Moreover, at the tree level in the perturbative expansion, there are only two connected tree diagrams, corresponding to the propagator and to the three vertex originating from the cubic interaction terms. For such theories it is derived here a set of BRST invariant observables, which lead to metric independent amplitudes. The vacuum expectation values of these observables can be computed exactly. From their expressions it is possible to isolate the Gauss linking number and an invariant of the Milnor type, which describes the topological relations among three or more closed curves.
\end{abstract}

*e-mail: fferrari@univ.szczecin.pl 


\section{Foreword}

In several situations it has been experimentally observed that the topological properties of certain physical systems may influence their behavior to a relevant extent. This is for instance the case of vortex structures in nematic liquid crystals [1] and in ${ }^{3} \mathrm{He}$ superfluids [2]. Other examples are provided by polymers [3] or by the lowest lying excitations of two-dimensional electron gases, which have topological non-trivial configurations at some filling fractions 顿. In the investigation of phenomena related to the presence of topological constraints in physical systems, the use of quantum or statistical mechanical models coupled to abelian Chern-Simons (C-S) field theories [5] has been particularly successful. One reason of this success is the fact that abelian models do not require a complex mathematical treatment as their non-abelian counterparts and thus their physical meaning is more transparent.

Motivated by applications in polymer physics [6, 6, 8] the aim of this work is the construction of topological field theories with non-trivial cubic interactions similar to those of non-abelian C-S field theories, but which still retain the simplicity of the abelian case. For this purpose, suitable candidates are C-S field theories with non-semisimple group of gauge symmetry. Roughly speaking, Lie algebras associated to non-semisimple groups contain non-trivial Abelian ideals, so that one could expect on this ground that at least part of the observables of these theories should have "Abelian" characteristics.

Chern-Simons field theories and, more in general, gauge field theories with non-semisimple groups of symmetry, have been already proposed in [9, 10]. Here it is picked up a particular class of such theories with the main property of being free from radiative corrections. Also at the tree level in the perturbative expansion several simplifications occur and it is possible to show that there are only two connected diagrams, the propagators and the three vertices corresponding to the fields' self-interactions. This situation is reminiscent to that of an Abelian field theory, in which there is just one connected diagram, namely the propagator. Most interestingly, the theories discussed in this work 
admit a set of observables which resemble Abelian Wilson loops and lead to metric independent amplitudes. With respect to standard Wilson loops, these observables contain extra terms, which are required to enforce BRST invariance. From their vacuum expectation values, which are computed exactly, it is possible to isolate a topological invariant, which describes the topological properties of three or more closed loops.

The material presented in this paper is divided as follows. In Section 2 a class of C-S field theories with non-semisimple group of symmetry is introduced, which consists in a set of abelian BF-models [12] coupled together by cubic interaction terms. The BRST quantization of these theories is discussed using the covariant gauge of Lorentz in order to fix gauge invariance. Further, it is shown that radiative corrections are absent and that there are only two connected Feynman diagrams at the tree level. The case of manifolds with non-trivial topology, in which zero modes may appear, is discussed in Section 3. It is shown that zero modes generate large gauge transformations which leave invariant the action and the equations of motion of the theories under consideration. In this way, it becomes possible to treat zero modes as gauge degrees of freedom and to gauge them away, as advocated in [13. In Section 4 a set of BRST invariant observables is derived and their vacuum expectation values is computed. Finally, the Conclusions and a possible physical application of the results contained in this work are presented in Section 5.

\section{Chern-Simons Field Theories with Non- Semisimple Group of Symmetry}

Let us consider a class of Chern-Simons field theories with action:

$$
S=\int_{M} \Omega_{i I} \epsilon^{\mu \nu \rho}\left(B_{\mu}^{I} \partial_{\nu} A_{\rho}^{i}+\frac{\lambda}{6} f_{j k}^{I} A_{\mu}^{i} A_{\nu}^{j} A_{\rho}^{k}\right)
$$

where $i, I=1, \ldots, N$ and $\Omega_{I i}$ denotes a non-degenerate bilinear form. Summation over repeated indices is everywhere understood. The theory is defined 
on a three dimensional manifold $M$ without boundary and equipped with an Euclidean metric. For simplicity, we assume for the moment that all the de Rham cohomology groups $H^{n}(M)$ are trivial, so that the problem of harmonic zero modes does not appear. We will discuss zero modes in the next Section.

The action (1) is invariant under the following gauge transformations:

$$
\begin{aligned}
A_{\mu}^{i} & \rightarrow A_{\mu}^{i}+\partial_{\mu} \eta^{i} \\
B_{\mu}^{I} & \rightarrow B_{\mu}^{I}+\partial_{\mu} \theta^{I}-\lambda f_{i j}^{I}\left(\frac{\eta^{i} \partial_{\mu} \eta^{j}}{2}+\eta^{i} A_{\mu}^{j}\right)
\end{aligned}
$$

for arbitrary functions $\eta^{i}$ and $\theta^{I}$. The above transformations correspond to a non-semisimple group of symmetry. The related generators $X_{i}$ and $H_{I}$ satisfy the following non-semisimple Lie algebra:

$$
\left[H_{I}, H_{J}\right]=\left[H_{I}, X_{j}\right]=0 \quad\left[X_{i}, X_{j}\right]=f_{i j}^{I} H_{I}
$$

with structure constants $f_{i j}^{I}$. This Lie algebra consists in an abelian Lie algebra $\mathfrak{g}$ with a central extension by an abelian group $\mathfrak{h}$. The generators of $\mathfrak{g}$ and $\mathfrak{h}$ are the $X_{i}$ and $H_{I}$ 's respectively. Let us denote with the symbols $\mathfrak{G}$ and $\mathfrak{H}$ respectively the associated abelian Lie groups. The matrix $\Omega_{I i}$ appearing in (更) is the generalization of the Killing form to the case of non-semisimple groups. Theories such as those discussed here have been already proposed in [9, 10]. A thorough discussion of their renormalization has been provided in [11]. Other applications of non-semisimple Lie algebras can be found in [15].

To eliminate the gauge freedom of the action (1), we introduce the covariant gauge conditions:

$$
\partial^{\mu} A_{\mu}^{i}=\partial^{\mu} B_{\mu}^{I}=0
$$

The theory can now be quantized using the procedure of BRST quantization. The BRST transformations associated to the gauge transformations (2) 3) are 
given by:

$$
\begin{array}{rlrl}
\delta A_{\mu}^{i} & =\partial_{\mu} c^{i} & \\
\delta B_{\mu}^{I} & =\partial_{\mu} \xi^{I}+\lambda f_{i j}^{I} A_{\mu}^{i} c^{j} \\
\delta \xi^{I} & =\frac{\lambda}{2} f_{i j}^{I} c^{i} c^{j} & \\
\delta c^{i} & =0 & \\
\delta \bar{c}_{i} & =i a_{i} & & \\
\delta \bar{\xi}_{I} & =i b_{I} & \delta b_{I}=0
\end{array}
$$

where $c^{i}, \xi^{I}$ and $\bar{c}_{i}, \bar{\xi}_{I}$ are anti-commuting ghosts, while $a_{i}, b_{I}$ are scalar fields. It is possible to verify that the transformations (6-11) are nilpotent, i. e. $\delta^{2}=0$.

At this point, one can write the expression of the gauge fixed BRST invariant action:

$$
S_{B R S T}=S+S_{g f}+S_{F P}
$$

where $S$ is given by Eq. (1), while the gauge fixing and Fadeev-Popov terms are respectively:

$$
\begin{gathered}
S_{g f}=i \int_{M} d^{3} x\left[a_{i} \partial^{\mu} A_{\mu}^{i}+b_{I} \partial^{\mu} B_{\mu}^{I}\right] \\
S_{F P}=\int_{M} d^{3} x\left[\partial_{\mu} \bar{c}_{i} \partial^{\mu} c^{i}+\partial^{\mu} \bar{\xi}_{I}\left(\partial_{\mu} \xi^{I}+\lambda f_{i j}^{I} A_{\mu}^{i} c^{j}\right)\right]
\end{gathered}
$$

The combination $S_{g f}+S_{F P}$ amounts to a BRST exact variation as expected:

$$
S_{g f}+S_{F P}=-\delta \int_{M}\left[\left(\bar{c}_{i} \partial^{\mu} A_{\mu}^{i}+\bar{\xi}_{I} \partial^{\mu} B_{\mu}^{I}\right]\right.
$$

Thus, the gauge fixing and Faddeev-Popov terms do not spoil the topological properties of the original action (11).

Let us note that the fields $B_{\mu}^{I}$ and $\xi^{I}$ play in (12) the role of pure Lagrange multipliers, which constrain the fields $A_{\mu}^{i}$ and $\bar{\xi}_{I}$ in such a way that all possible radiative corrections vanish identically. In particular, the interaction term in the ghost action (14) disappears after an integration over the fields $\xi^{I}$, which gives as a result the constraints

$$
\partial_{\mu} \partial^{\mu} \bar{\xi}_{I}=0
$$


Choosing suitable boundary conditions for which the $\bar{\xi}_{I}$ 's do not diverge at infinity, the above equation is satisfied only for constant fields $\bar{\xi}_{I}$. The integration over the fields $B_{\mu}^{I}$ leads instead to the flatness conditions:

$$
\Omega_{I i} \epsilon^{\mu \nu \rho} \partial_{\nu} A_{\rho}^{i}=0
$$

The above equations determine the transverse components of the fields $A_{\rho}^{i}$, while the longitudinal components are fixed by the gauge fixing (13).

Since radiative corrections are absent, the theory (12) is purely classical. Thus, contrarily to what happens for instance in the case of Chern-Simons theories with gauge group $S U(N)$, there is no rescaling of the Chern-Simons coupling constants, which here have been set equal to one. Also at the classical level several simplifications occurs and in practice the theory admits only the two connected Feynman diagrams shown in Fig. 8. These diagrams correspond to the field propagators and to the three-vertex associated to the cubic interaction term present in Eq. (12). Higher order tree diagrams, which could in principle be constructed by contracting together the legs of many three-vertices, are actually ruled out due to the off-diagonal structure of the propagators, which forbids any self-interaction among the fields $A_{\mu}^{i}$.

Due to the fact that the theory is purely classical, its partition function can be exactly derived once the classical solutions of the field equations are known. With a simple integration it is possible to eliminate the fields $B_{\mu}^{I}$ and the ghosts. As an upshot, one obtains the constraints (16 17). These constraints and the gauge fixing relations are enough to determine uniquely the remaining fields. If the theory is defined for instance on a manifold with flat metric, the solutions of the constraints (17) are simply $A_{\mu}^{i}=0$, so that the partition function is the trivial one.

\section{The Zero Mode Problem}

In this Section we consider the case in which the fields admit non-trivial classical configurations, the so-called harmonic zero modes. We can ignore possible zero 
modes in the sectors of the ghost fields and of the Lagrange multipliers $a_{i}, b_{I}$, because these zero modes are not used in the gauge fixing procedure. We are thus left only with the zero modes of the fields $A_{\mu}^{i}, B_{\mu}^{I}$, which we denote with the symbols $\alpha_{\mu}^{i}$ and $\beta_{\mu}^{I}$ respectively. From the action (11) one finds the relevant equations of motion which define $\alpha_{\mu}^{i}$ and $\beta_{\mu}^{I}$ :

$$
\begin{aligned}
\epsilon^{\mu \nu \rho} \partial_{\nu} A_{\rho}^{i} & =0 \\
\epsilon^{\mu \nu \rho} \partial_{\nu} B_{\rho}^{I} & +\frac{\lambda}{2} \epsilon^{\mu \nu \rho} f_{j k}^{I} A_{\nu}^{j} A_{\rho}^{k}=0
\end{aligned}
$$

The general solution of Eq. (18) is

$$
A_{\mu}^{i}=\alpha_{\mu}^{i}+\partial_{\mu} \eta^{i}
$$

where $\partial_{\mu} \eta^{i}$ is an exact differential, while $\alpha_{\mu}^{i}$ is a non-trivial abelian flat connection corresponding to the abelian subgroup of the underlying gauge group. Let us consider now Eq. (19). This relation can be rewritten as follows:

$$
\epsilon^{\mu \nu \rho} \partial_{\nu} B_{\rho}^{I}=J^{I \mu}
$$

with the current $J^{I \mu}$ given by:

$$
J^{I \mu}=-\frac{\lambda}{2} \epsilon^{\mu \nu \rho} f_{j k}^{I} A_{\nu}^{j} A_{\rho}^{k}
$$

Eq. (18) implies that $J^{I \mu}$ is purely transverse, i. e. $\partial_{\mu} J^{I \mu}=0$. Using this fact, it is possible to show that Eq. (19) is solved by:

$$
B_{\mu}^{I}=\int_{M} d^{3} y G_{\mu \nu}(x, y) J^{I \nu}(y)+\beta_{\mu}^{I}+\partial_{\mu} \eta^{I}
$$

where $G_{\mu \nu}(x, y)$ is the propagator of the theory in the Lorentz gauge (5) and $\beta_{\mu}^{I}$ is a non-trivial flat connection satisfying the flatness condition $\epsilon^{\mu \nu \rho} \partial_{\nu} \beta_{\rho}^{I}=0$. We remember that the fields $B_{\mu}^{I}$ are pure Lagrange multipliers imposing the constraints (18) on the fields $A_{\mu}^{i}$. It is easy to check that the presence or not of the term $\beta_{\rho}^{I}$ does not affect these constraints nor the other equations of motion, so that one can put $\beta_{\mu}^{I}=0$ without any loss of generality.

A possible strategy to treat harmonic zero modes is to consider them as gauge degrees of freedom and to gauge them away using BRST techniques. 
This approach has been proposed by Polyakov in [13] and further developed in [16]. An application to the $B F$-systems can be found in [17]. In order to check if it is possible to translate the zero mode problem in a gauge fixing problem also in the present case, the crucial point is to verify the invariance of the theory (11) under large gauge transformations. As a matter of fact, the $\alpha_{\mu}^{i}$ 's generate large gauge transformations, consisting in multivalued mapping of the manifold $M$ onto the elements of the abelian group which corresponds to the Lie algebra $\mathfrak{G}$ defined after Eq. (伊).

To express the large gauge transformations acting on the fields in a closed form, it is convenient to introduce potentials $\Lambda^{i}$ such that:

$$
\partial_{\mu} \Lambda^{i}=\alpha_{\mu}^{i}
$$

These potentials, which will be in general multivalued on the manifold $M$, are the analogous of the functions $\eta^{i}$ appearing in the gauge transformations (2-3). In terms of the $\Lambda^{i}$ s, the large gauge transformations induced by the harmonic modes $\alpha_{\mu}^{i}$ are given by:

$$
\begin{aligned}
A_{\mu}^{i} & \rightarrow A_{\mu}^{i}+\partial_{\mu} \Lambda^{i} \\
B_{\mu}^{I} & \rightarrow B_{\mu}^{I}-\lambda f_{i j}^{I}\left(\frac{\Lambda^{i} \partial_{\mu} \Lambda^{j}}{2}+\Lambda^{i} A_{\mu}^{j}\right)
\end{aligned}
$$

Actually, the full transformations of the fields $B_{\mu}^{I}$ are not necessary in order to discuss the gauge invariance of the action $S$ and of the equations of motion. As a matter of fact, apart from a total derivative, the action (1) can be rewritten as follows:

$$
S=\int_{M} d^{3} x \Omega_{I i} \epsilon^{\mu \nu \rho} A_{\mu}^{i}\left[\partial_{\nu} B_{\rho}^{I}+\frac{\lambda}{6} f_{j k}^{I} A_{\nu}^{j} A_{\rho}^{k}\right]
$$

Thus, only the transformations of the pseudo-tensors $\epsilon^{\mu \nu \rho} \partial_{\nu} B_{\rho}^{I}$ are needed:

$$
\begin{aligned}
\epsilon^{\mu \nu \rho} \partial_{\nu} B_{\rho}^{I} & \rightarrow \epsilon^{\mu \nu \rho} \partial_{\nu} B_{\rho}^{I} \\
& -\frac{\lambda}{2} \epsilon^{\mu \nu \rho} f_{j k}^{I}\left(\partial_{\nu} \Lambda^{j} \partial_{\rho} \Lambda^{k}+2 \Lambda^{j} \partial_{\nu} A_{\rho}^{k}+2 \partial_{\nu} \Lambda^{j} A_{\rho}^{k}\right)
\end{aligned}
$$

At this point we are ready to perform a large gauge transformation of the kind (25) in the action $S$. Since the right hand side of Eq. (28) has an 
explicit dependence on the potentials $\Lambda^{i}$, the gauge transformed action will contain multivalued contributions. However, it is easy to prove that all these contributions vanish identically due to the following identities, valid up to total derivative terms which are irrelevant on a manifold without boundary:

$$
\begin{aligned}
\int_{M} d^{3} x \Omega_{I i} \epsilon^{\mu \nu \rho} f_{j k}^{I} A_{\mu}^{i} \partial_{\rho} A_{\nu}^{j} \Lambda^{k} & =-\frac{1}{2} \int_{M} d^{3} x \Omega_{I i} \epsilon^{\mu \nu \rho} f_{j k}^{I} A_{\mu}^{i} A_{\nu}^{j} \alpha_{\rho}^{k} \\
\int_{M} d^{3} x \Omega_{I i} \epsilon^{\mu \nu \rho} f_{j k}^{I} \alpha_{\mu}^{i} \partial_{\rho} A_{\nu}^{j} \Lambda^{k} & =-\int_{M} d^{3} x \Omega_{I i} \epsilon^{\mu \nu \rho} f_{j k}^{I} \alpha_{\mu}^{i} A_{\nu}^{j} \alpha_{\rho}^{k}
\end{aligned}
$$

Another important identity, which follows from the fact that the $\alpha_{\mu}^{i}$ 's satisfy the classical equations of motion (18), is:

$$
\int_{M} d^{3} x \Omega_{I i} \epsilon^{\mu \nu \rho} f_{j k}^{I} \alpha_{\mu}^{i} \alpha_{\nu}^{j} \alpha_{\rho}^{k}=\int_{M} d^{3} x \Omega_{I i} \epsilon^{\mu \nu \rho} f_{j k}^{I} \partial_{\mu} \Lambda^{i} \partial_{\nu} \Lambda^{j} \partial_{\rho} \Lambda^{k}=0
$$

With the help of the relations (29-31) it is possible to verify the invariance of the action $S$ under large gauge transformations as desired.

One can also check that the gauge transformations (25) and 28) preserve the form of the equations of motion (18 19). After a gauge transformation, in fact, one obtains the following result:

$$
\begin{aligned}
\epsilon^{\mu \nu \rho} \partial_{\nu} A_{\rho}^{i} & =0 \\
\epsilon^{\mu \nu \rho} \partial_{\nu} B_{\rho}^{I} & +\frac{\lambda}{2} \epsilon^{\mu \nu \rho} f_{j k}^{I}\left(A_{\nu}^{j} A_{\rho}^{k}-2 \partial_{\nu} A_{\rho}^{k} \Lambda^{j}\right)=0
\end{aligned}
$$

The spurious term proportional to $\Lambda^{j}$ in Eq. (33) vanishes identically due to Eq. (32).

\section{Observables and Wilson Loop-like ampli- tudes}

Good observables of a topological field theory should be BRST invariant and lead to vacuum expectation values which are metric independent.

To derive a set of observables for the theory under consideration, we first observe that the following quantity is invariant under the BRST transformations 
(6-11):

$$
\begin{aligned}
& T^{I}(\Gamma)=\oint_{\Gamma} d x^{\mu} B_{\mu}^{I}+\frac{\lambda}{4 \pi} f_{i j}^{I} \oint_{\Gamma} d x^{\mu} A_{\mu}^{i} \int d^{3} y \frac{1}{|x-y|} \partial_{y}^{\rho} A_{\rho}^{j}(y) \\
& +\frac{\lambda}{2(4 \pi)^{2}} f_{i j}^{I} \oint_{\Gamma} d x^{\mu} \int d^{3} y\left(\partial_{\mu}^{x} \frac{1}{|x-y|}\right) \partial_{y}^{\rho} A_{\rho}^{i}(y) \int d^{3} z \frac{1}{|x-z|} \partial_{z}^{\sigma} A_{\sigma}^{j}(z)
\end{aligned}
$$

In the above formula as well as in the rest of this paper, it has been assumed for simplicity that the manifold $M$ coincides with the three dimensional euclidean space $\mathbf{R}^{3}$. The form of $T^{I}(\Gamma)$ has been obtained starting from the line integral $\oint_{\Gamma} d x^{\mu} B_{\mu}^{I}$ and adding suitable terms in order to make it gauge and BRST invariant. At this point, for $M$ loops $\Gamma^{a}, a=1, \ldots, M$, it is possible to write down analogs of the holonomic connections as follows:

$$
W(\bar{C})=e^{i C_{a I} T^{I}\left(\Gamma^{a}\right)}
$$

where $\bar{C}$ is a matrix having as elements constant parameters $C_{a I}$.

We note that $W(\bar{C})$ is of the form:

$$
W(\bar{C})=\exp \left[i C^{a I} \oint_{\Gamma_{a}} d x^{\mu} B_{\mu}^{I}+\int d^{3} x \chi_{i}(x) \partial^{\mu} A_{\mu}^{i}(x)\right]
$$

All contributions coming from the various line integrals which are present in the right hand side of Eq. (34) are now contained in the scalars $\chi_{i}(x)$. As a consequence of Eq. (36), even if $W(\bar{C})$ is manifestly metric dependent, a metric variation of this observable can always be compensated by a shift of the Lagrange multipliers $a_{i}$ which impose the gauge condition in the action (12). Thus, the vacuum expectation values of the operators $W(\bar{C})$ lead to metric independent amplitudes as required.

When computing the vacuum expectation value $\langle W(\bar{C})\rangle$ of the operator (34), several simplifications occur. One reason is that the fields $A_{\mu}^{i}$ are unaffected by the cubic interactions present in the action (12), because they can be contracted only with the fields $B_{\mu}^{I}$ due to the off-diagonal form of the kinetic terms. On the second hand, as already mentioned, the fields $B_{\mu}^{I}$ behave as abelian fields and act as Lagrange multipliers, which impose the conditions:

$$
\Omega_{I i} \epsilon^{\mu \nu \rho} \partial_{\nu} A_{\rho}^{i}(x)+\sum_{a=1}^{M} C_{I a} \oint_{\Gamma^{a}} d y^{\mu} \delta(x-y)=0
$$


The solution of the above equation is given by:

$$
A_{\mu}^{(c l) i}(x)=\left(\Omega^{-1}\right)^{i I} C_{I a} \mathcal{B}_{\mu}^{a}(x)
$$

with

$$
\mathcal{B}_{\mu}^{a}(x)=-\frac{\epsilon_{\mu \sigma \tau}}{4 \pi} \oint_{\Gamma^{a}} d x^{\sigma} \partial^{\tau} \frac{1}{|x-y|}
$$

Let us note that $A_{\mu}^{(c l) i}(x)$ is a purely transverse vector field because the longitudinal components have been fixed to zero by the gauge condition (5). At this point, it is possible to evaluate the explicit expression of $\langle W(\bar{C})\rangle$ using the saddle point evaluation method. After some calculations one finds:

$$
\langle W(\bar{C})\rangle=\exp \left[\frac{i \lambda}{6} \int d^{3} x l_{a b c} \epsilon^{\mu \nu \rho} \mathcal{B}_{\mu}^{a}(x) \mathcal{B}_{\nu}^{b}(x) \mathcal{B}_{\rho}^{c}(x)\right]
$$

where

$$
l_{a b c}=\left(\left(\Omega^{-1}\right)^{j J}\left(\Omega^{-1}\right)^{k K} C_{I a} C_{J b} C_{K c} f_{j k}^{I}\right.
$$

From Eq. (40) it turns out that the vacuum expectation values of the operators $W(\bar{C})$ deliver essentially a single topological invariant which is given by:

$$
\mathcal{H}=\int d^{3} x l_{a b c} \epsilon^{\mu \nu \rho} \mathcal{B}_{\mu}^{a}(x) \mathcal{B}_{\nu}^{b}(x) \mathcal{B}_{\rho}^{c}(x)
$$

Another topological invariant, namely the Gauss linking number, can be obtained by considering also amplitudes containing Abelian holonomic connections of the $A_{\mu}^{i}$ fields. Abelian connections are sufficient to grant BRST invariance in this case due to the simplicity of the BRST transformations (6) of the fields $A_{\mu}^{i}$.

\section{Conclusions}

In this work we have investigated a class of topological field theories having the property that its perturbative series contains only the finite set of Feynman diagrams given in Fig. 8. These theories are exactly solvable and, besides the Gauss link invariant which is typical of Abelian C-S field theories, produce the topological invariant $\mathcal{H}$ of Eq. (42). The fact that a non-Abelian Chern-Simons 
field theory can sustain Abelian observables like the $W(\bar{C})$ of Eq. (34) is related to the presence of a non-trivial Abelian ideal in the gauge group of symmetry.

It is interesting to consider the above results from the point of view of possible applications to the statistical mechanics of random walks. Let $\Gamma^{1}, \ldots, \Gamma^{M}$ be a set of $M$ closed random walks, interacting together via the topological potential (42). The partition function of this system coincides with the sum over all possible configurations of the trajectories $\Gamma^{1}, \ldots, \Gamma^{M}$ :

$$
Z=\int \mathcal{D} \mathbf{r}_{1}\left(s_{1}\right) \ldots \mathcal{D} \mathbf{r}_{M}\left(s_{M}\right) \exp \left[\sum_{a=1}^{M} \oint_{\Gamma^{a}} \dot{\mathbf{r}}_{a}^{2}+\mathcal{H}\left(\mathbf{r}_{1}, \ldots, \mathbf{r}_{M}\right)\right]
$$

where $\mathbf{r}_{a}\left(s_{a}\right), a=1, \ldots, M$ are curves described in the space by the paths $\Gamma^{a}$ and parametrized by means of their arc-lengths $s_{a}$. At this point the relation

(40) may be interpreted as the analog of an Hubbard-Stratonovich transformation [6], which decouples the trajectories $\Gamma^{a}$ in the partition function $Z$. Such transformation simplifies the task of performing the path integration in Eq. (43), which is otherwise very complicated due to the presence of the topological term $\mathcal{H}\left(\mathbf{r}_{1}, \ldots, \mathbf{r}_{M}\right)$. Other applications can be in the study of the fractional quantum Hall effect 18, 19.

To conclude, one should mention that the idea of constructing a topological field theory with a finite number of Feynman diagrams has already been realized following a different route. This is the so-called Rozansky-Witten topological sigma model, which delivers topological invariants of the Milnor type of its hyper-Kähler target space [20]. Interesting new developments in this direction have been presented in [21, 22].

\section{Acknowledgments}

I wish to thank heartily the referee of this paper, G. Thompson, whose contribution has led to considerable improvements with respect to the original version. In particular, I am grateful to G. Thompson for pointing out that the theory in Eq. (11) is related to a Chern-Simons field theory with non-semisimple group of gauge symmetry and for suggesting Eqs. (2-3), which provide the full 
set of gauge transformations of the theory. I am also grateful to G. Thompson for having written a very enlightening referee report on this paper and for his interest in my work. Finally, I wish to thank him for drawing my attention to the stimulating problem of the treatment of harmonic zero modes.

\section{References}

[1] M. J. Bowick, L. Chander, E. A. Schiff and A. M. Srivastava, Science 263 (1994), 943.

[2] C. Bäuerle et al., Nature 382 (1996), 332; V. M. H. Ruutu et al., Nature 382 (1996), 334.

[3] E. Wasserman, Jour. Am. Chem. Soc. 82 (1960), 4433.

[4] D. R. Leadly et al., Phys. Rev. Lett. 79 (1997), 4246.

[5] A. S. Schwarz, Lett. Math. Phys. 2 (1978), 247; S. Deser, R. Jackiw and S. Templeton, Phys. Rev. Lett. 48 (1982), 975; S. Deser, R. Jackiw and S. Templeton, Annals Phys. 140 (1982), 372; C. R. Hagen, Annals Phys. 157 (1984), 342.

[6] F. Ferrari, Annalen der Physik (Leipzig) 11 (2002) 4, 255-290.

[7] A. L. Kholodenko and T. A. Vilgis, Phys. Rep. 298 (1998), 251.

[8] H. Kleinert, Path Integrals, (World Scientific Publishing, 2nd Ed., Singapore, 1995).

[9] E. Witten, Nucl. Phys. B311 (1988/89), 46.

[10] A. A. Tseytlin, Nucl. Phys. B 450 (1995), 231 arXiv:hep-th/9505129.

[11] P. A. Grassi, Nucl. Phys. B 560 (1999), 499, arXiv:hep-th/9908188.

[12] G. Horowitz, Comm. Math. Phys. 125 (1989), 417; A. S. Schwartz, Comm. Math. Phys. 67(1979), 1; M. Blau and G. Thompson, Ann. Phys. (NY) 205 (1991), 130.

[13] A. M. Polyakov, Nucl. Phys. B120 (1977), 429. 
[14] S. F. Edwards, Proc. Phys. Soc. 91 (1967), 513; J. Phys. A1 (1968), 15.

[15] C. Nappi and E. Witten, Phys. Lett. B293 (1992), 309; J. M. FigueroaO'Farrill and S. Stanciu, Phys. Lett. B327 (1994), 40; D. Olive, E. Rabinovici and A. Schwimmer, Phys. Lett. B321 (1994), 361; E. Kiritsis, C. Kounnas and D. Lüst, Phys. Lett. B331 (1994), 321.

[16] D. Amati and A. Rouet, Nuovo Cim. 50A (1979), 265; O. Babelon, Nucl. Phys. B131 (1977), 519.

[17] M. Blau and G. Thompson, Annals Phys. 205 (1991), 130.

[18] F. Wilczek, Fractional Statistics and Anyon Superconductivity, (World Scientific, New Jersey 1990), Ch. 9; X. G. Wen, Phys. Rev. B43 (1991), 11025.

[19] F. A. Bais, P. van Driel and M. de Wild Propitius, Nucl. Phys. B393 (1993), 547, arXiv:hep-th/9203047.

[20] L. Rozansky and E. Witten, Selecta Math. 3 (1997), 401-458, hepth/9612216.

[21] N. Habegger and G. Thompson, "The Universal Perturbative Quantum 3Manifold Invariant, Rozansky-Witten invariants and the Generalized Casson Invariant", math.gt/9911049.

[22] B. Broda and M. Bakalarska, Phys. Lett. B472 (2000), 341, hepth/9911130; B. Broda, "3-dimensional topological $\sigma$-model", hepth/0201006. 


\section{$7 \quad$ Figure Captions}

Fig. 1 Feynman rules corresponding to the action (12). Dashed lines propagate $B_{\mu}^{I}$ fields while solid lines are associated to $A_{\mu}^{i}$ fields. 


\section{Figures}

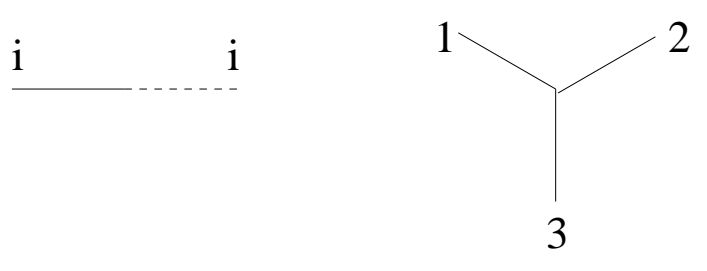

Fig. 1 